\title{
Self-Compassion as a Resource in the Self-Stigma Process of Overweight and Obese Individuals
}

\author{
Anja Hilbert ${ }^{a, b} \quad$ Elmar Braehler ${ }^{b, c}$ Ricarda Schmidtt ${ }^{a, b} \quad$ Bernd Löwe $^{d}$ \\ Winfried Häusere, ${ }^{f}$ Markus Zenger ${ }^{b}$ \\ antegrated Research and Treatment Center Adiposity Diseases, University of Leipzig, \\ Leipzig, Germany; ${ }^{b}$ Department of Medical Psychology and Medical Sociology, University \\ of Leipzig, Leipzig, Germany; ' Department of Psychosomatic Medicine and Psychotherapy, \\ University Medical Center of the Johannes Gutenberg-University, Mainz, Germany; \\ ${ }^{\mathrm{d} D e p a r t m e n t}$ of Psychosomatic Medicine and Psychotherapy, University Medical Center

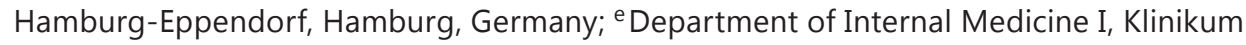 \\ Saarbrücken, Saarbrücken, Germany; ${ }^{\mathrm{f}}$ Department of Psychosomatic Medicine and \\ Psychotherapy, Technische Universität München, Munich, Germany
}

Key Words

Stigma · Weight bias · Self-compassion · Depression · Somatic symptoms · Health · Quality of life

\begin{abstract}
Objective: Self-stigma in overweight and obese individuals has strong associations with impairment in mental and global health. This study sought to explore self-compassion as a psychological resource in the self-stigma process. Methods: In a 2012 representative German population survey of $\mathrm{N}=1,158$ overweight and obese individuals, self-compassion was examined as a mediator between self-stigma and mental and physical health outcomes, including BMI $\left(\mathrm{kg} / \mathrm{m}^{2}\right)$, using structural equation modeling and controlling for sociodemographic factors. Results: Psychological variables were assessed using validated self-report questionnaires. Self-compassion partially mediated the relationships between self-stigma and depression, somatic symptoms, and health status / quality of life, lowering the predictive effect of self-stigma on the outcomes by approximately one-third. In contrast, self-compassion, because it was unrelated to BMI, did not mediate the association between self-stigma and BMI. Conclusion: Self-compassion has the potential to act as a buffer against the mental and global health detriments of self-stigma in overweight and obesity and could thus represent a target for interventions to reduce self-stigma and prevent these health impairments. In order to influence the association between self-stigma and BMI, self-compassion should conceptually be linked to weight management.


Hilbert et al.: Self-Compassion as a Resource in the Self-Stigma Process of Overweight and Obese Individuals

\section{Introduction}

Social values give clear standards of an individual's physical appearance and societal beliefs about its controllability [1]. Holding stigmatized attributes refers to being socially devaluated or rejected because of traits and attitudes that are deeply discrediting [2]. Overweight and obesity are such powerful social markers that both imply weight deviance and personal failure of weight control [3]. Indeed, overweight and obese individuals are publicly exposed to pervasive weight bias, including negative stereotypes and prejudice such as attributions of blame or incompetence that may extend to actual discrimination in multiple domains of life [4].

Self-stigma emerges when stigmatized individuals internalize these stereotypes, show negative emotional reactions, and discriminate themselves [5, 6]. Self-stigma, thus, involves negative beliefs about the self, strong negative feelings, for example of self-hate and shame, as well as putting oneself at a disadvantage, for example, through social withdrawal. In fact, obese individuals often show signs of self-stigma, devalue themselves, have low weightrelated self-esteem, and feel ashamed and hate themselves because of their weight [7-10]. Consequently, self-stigma is strongly related to psychopathology, social and behavioral problems, and impaired quality of life in obese individuals [11-16]. The explanatory power of self-stigma exceeds that of stigmatizing attitudes, experiences of weight-related discrimination, and BMI $\left(\mathrm{kg} / \mathrm{m}^{2}\right)$. However, the associations with BMI are inconsistent, and one study did not show prognostic significance for weight loss [12].

Research has only begun to understand the self-stigma process and to explain how selfstigma impacts overweight and obese individuals [11]. Largely unknown is which psychological resources may help to alleviate the adverse consequences of weight self-stigma. Evidence from other stigmatized conditions indicates that stigmatized individuals may engage in a variety of self-protective cognitive strategies $[17,18]$, with self-compassion being one of these potential resources. Self-compassion is defined as a healthy attitude toward oneself with regard to one's failures and inadequacies [19]. In a student sample, self-compassion moderated the positive association between BMI and eating disorder psychopathology [20]; a higher BMI was only related to greater eating disorder psychopathology among women with lower levels of self-compassion [20]. Results from other nonobese samples indicated that self-compassion shows substantial cross-sectional associations with lower general (e.g., depression, anxiety, stress, pain) [21,22] and eating disorder psychopathology [23-26], and was found to be predictive of greater weight loss [27]. In this context, self-compassion has been assumed to act as a key mechanism explaining the efficacy of mindfulness-based interventions, increasingly examined for the prevention and treatment of obesity [28, 29]. Whether self-compassion represents a resource in the psychopathology-prone self-stigma process in overweight and obese individuals, however, warrants further investigation. This study sought to examine the impact of self-compassion as a mediator between self-stigma and mental and physical health outcomes, including BMI.

\section{Material and Methods}

\section{Recruitment and Sample}

Based on a 2012 representative survey of the German population, the sample was selected with the assistance of the Independent Service for Surveys, Methods and Analyses (USUMA Berlin) using a three-stage random-route-assisted sampling procedure (for full methodological detail see [11]). Inclusion criteria were age $\geq 14$ years and the ability to read and understand German. Of the 4,436 valid addresses, 2,510 partici- 
Hilbert et al.: Self-Compassion as a Resource in the Self-Stigma Process of Overweight and Obese Individuals

pants were included in the total sample (response rate 56.6\%). For the purpose of this study, participants with a BMI $\geq 25.0 \mathrm{~kg} / \mathrm{m}^{2}$ were selected, leaving a study sample of $\mathrm{N}=1,158$ overweight or obese men and women. The 629 men (54.3\%) and 529 women (45.7\%) were on average $53.56 \pm 16.22$ years old, with a majority having obtained less than 12 years of education $(990 / 1,158,85.5 \%)$. Based on self-reported height and weight, 931 (80.4\%) participants were classified as overweight $\left(25.0 \geq \mathrm{BMI}<30.0 \mathrm{~kg} / \mathrm{m}^{2}\right)$, and 227 $(19.6 \%)$ as obese (BMI $\left.\geq 30.0 \mathrm{~kg} / \mathrm{m}^{2}\right)$.

The Ethics Committee of the University of Leipzig approved this study (Approval No. 092-12-05032012). Accordingly, participants provided their oral informed consent prior to assessment (for minor participants, oral informed consent was also obtained from one parent). Oral consent is common in survey research in Germany. The ethical guidelines of the International Code of Marketing and Social Research Practice by the International Chamber of Commerce and the European Society for Opinion and Marketing Research were followed.

\section{Measures}

\section{Predictor Variable}

A validated self-report-questionnaire was used to assess self-stigma, the Weight Bias Internalization Scale (WBIS) $[13,15]$. The 10-item scale measures the degree to which a respondent directs negative societal beliefs about obesity toward the self [13], e.g., 'I hate myself for being overweight', 1 = 'strongly disagree' to 7 = 'strongly agree'. In the WBIS, self-stigma is operationalized as weight-related self-criticism, including self-hate, self-blame, and feelings of inadequacy. Higher mean scores indicate greater self-stigma $(\alpha=0.91)$.

\section{Mediator Variable}

The Self-Compassion Scale (SCS) [19, 30] was used to assess self-compassion. The SCS is a 26-item selfreport measure of compassionate responding to oneself, with 6 subscales measuring 3 components of selfcompassion (self-kindness vs. self-judgement, common humanity vs. isolation, mindfulness vs. over-identification), e.g., 'I try to be understanding and patient towards those aspects of my personality I don't like', $1=$ 'almost never' to 5 = 'almost always'. Higher mean subscale scores indicate higher levels of positive selfcompassion (comprising the subscales self-kindness, common humanity, mindfulness) and lower levels of self-critical judgment (comprising the subscales self-judgment, isolation, over-identification), respectively. A higher SCS total mean score indicates a higher level of self-compassion $(\alpha=0.92)$.

\section{Outcome Variables}

The 8-item Somatic Symptom Score-8 (SSS-8) [31] was used to assess somatic symptom strain, e.g., 'During the past 7 days, how much have you been bothered by any of the following problems?', $0=$ 'not at all' to 4 = 'very much'. A higher sum score indicates more somatic symptoms $(\alpha=0.81)$.

Depressive symptoms such as dysphoria (0-3, with higher scores representing greater severity) were assessed through the 7-item Beck Depression Inventory for Primary Care (BDI-PC) [32, 33]. A higher sum score indicates more depressive symptomatology $(\alpha=0.86)$.

Health status / quality of life was measured through a 2-item scale of the European Organization for Research and Treatment of Cancer Quality of Life Questionnaire - Core 30 (EORTC QLQ-C30) [34, 35], e.g., 'How would you rate your overall health during the past week?', 1 = 'very poor' to 7 = 'excellent'. Raw mean scores were transformed to $0-100$, with higher scores indicating higher quality of life.

\section{Data Analysis}

First, descriptive statistics and Pearson's zero-order correlations were computed between predictor (WBIS, SCS total score) and global health variables (BDI-PC, SSS-8, EORTC QLQ-C30, BMI). Second, for exploratory analyses on SCS subscales (see below), collinearity between WBIS and SCS subscales was analyzed prior to mediation analysis using Pearson's correlations.

Using structural equation modeling (IBM ${ }^{\circledR}$ SPSS AMOS $^{\circledR}$ version 20.0; Chicago, IL, USA), direct associations between self-stigma and outcome variables (depression, somatic symptoms, health status / quality of life, BMI) were examined, controlling for socioeconomic variables (gender, age, education) in Model 1. In Model 2, self-compassion was examined as a mediator of the relationship between self-stigma and outcome variables. In case of significance, post-hoc analyses were used to identify which of the 6 SCS subscales 
Hilbert et al.: Self-Compassion as a Resource in the Self-Stigma Process of Overweight and Obese Individuals

Table 1. Descriptive statistics and correlation matrix of study variables $(\mathrm{N}=1,158)$

\begin{tabular}{|c|c|c|c|c|c|c|c|c|c|}
\hline & & M & SD & 1 & 2 & 3 & 4 & 5 & 6 \\
\hline 1 & WBIS & 2.65 & 1.19 & 1 & & & & & \\
\hline 2 & SCS & 3.35 & 0.43 & -0.31 & 1 & & & & \\
\hline 3 & BDI-PC & 1.36 & 2.24 & 0.28 & -0.43 & 1 & & & \\
\hline 4 & SSS-8 & 3.66 & 3.98 & 0.27 & -0.30 & 0.56 & 1 & & \\
\hline 5 & EORTC QLQ-C30 & 72.52 & 20.28 & -0.24 & 0.28 & -0.49 & -0.64 & 1 & \\
\hline 6 & BMI & 28.22 & 3.66 & 0.21 & -0.06 & 0.16 & 0.19 & -0.20 & 1 \\
\hline
\end{tabular}

WBIS = Weight Bias Internalization Scale (1-7*, scores indicating less favorable conditions are asterisked); BDI-PC = Beck Depression Inventory for Primary Care (0-21*); SCS = Self-Compassion Scale (1*-5); SSS-8 = Somatic Symptom Score-8 (0-32*); EORTC QLQ-C30 = European Organization for Research and Treatment of Cancer Quality of Life Questionnaire - Core $30(0 *-100)$.

Table 2. Goodness-of-fit indices of structural equation modeling $(\mathrm{N}=1,158)$

\begin{tabular}{llllllllll}
\hline & $\chi^{2}$ & df & CMIN/DF & CFI & GFI & RMSEA & TLI & NFI & SRMR \\
\hline Model 1 $^{\mathrm{a}}$ & 30.110 & 12 & 2.509 & 0.988 & 0.994 & 0.036 & 0.973 & 0.981 & 0.032 \\
Model 2 $^{\mathrm{b}}$ & 40.227 & 16 & 2.514 & 0.987 & 0.992 & 0.036 & 0.971 & 0.979 & 0.033 \\
\hline
\end{tabular}

$\mathrm{df}=$ Degrees of freedom; CMIN/DF = minimum discrepancy, divided by degrees of freedom; CFI = comparative-fit index; GFI = goodness-of-fit index; RMSEA = root mean square error of approximation; TLI = Tucker-Lewis index; NFI = normed fit index; SRMR = standardized root mean square residual.

${ }^{a}$ Model 1 includes prediction effects of self-stigma on health outcomes.

${ }^{\mathrm{b}}$ Model 2 includes self-compassion as mediator between self-stigma and health outcomes.

accounted for the mediation effects. In these analyses, SCS total score was replaced by the SCS subscales in separate steps.

The following indices were determined for evaluation of model fit: $\chi^{2}$ test statistics; the minimum discrepancy, divided by its degrees of freedom (CMIN/DF); the goodness-of-fit-index (GFI); the comparativefit index (CFI); the Tucker-Lewis-Index (TLI); the normed fit index (NFI); the root mean square error of approximation (RMSEA); and the standardized root mean square residual (SRMR). A non-significant $\chi^{2}$ value indicates that the model is compatible with the data. Further, good model fit is indicated with a ratio CMIN/ DF close to 2; with GFI, CFI, TLI, and NFI > 0.95; and with RMSEA and SRMR $<0.08$ [36]. Standardized regression weights were interpreted as indicative of small $(<-0.30)$, medium $(<-0.50)$, or large $(\geq-0.50)$ effects [37].

\section{Results}

Zero-order correlations between all study variables revealed that self-stigma (WBIS) was significantly associated with self-compassion (SCS) and all global health outcomes (small to medium effects; table 1). Self-compassion showed medium- to large-size associations with global health variables except for BMI (small effect).

Testing for collinearity between predictor variables assured orthogonality among selfstigma (WBIS) and all SCS subscales. Pearson's correlations indicated medium negative associations between WBIS and negative SCS subscales (self-judgement, isolation, over-identification; $-0.33 \leq r \leq-0.25$ ), while associations for WBIS and positive SCS subscales were closeto-zero (self-kindness, common humanity, mindfulness; $0.01 \leq \mathrm{r} \leq 0.09$ ). 
Hilbert et al.: Self-Compassion as a Resource in the Self-Stigma Process of Overweight and Obese Individuals

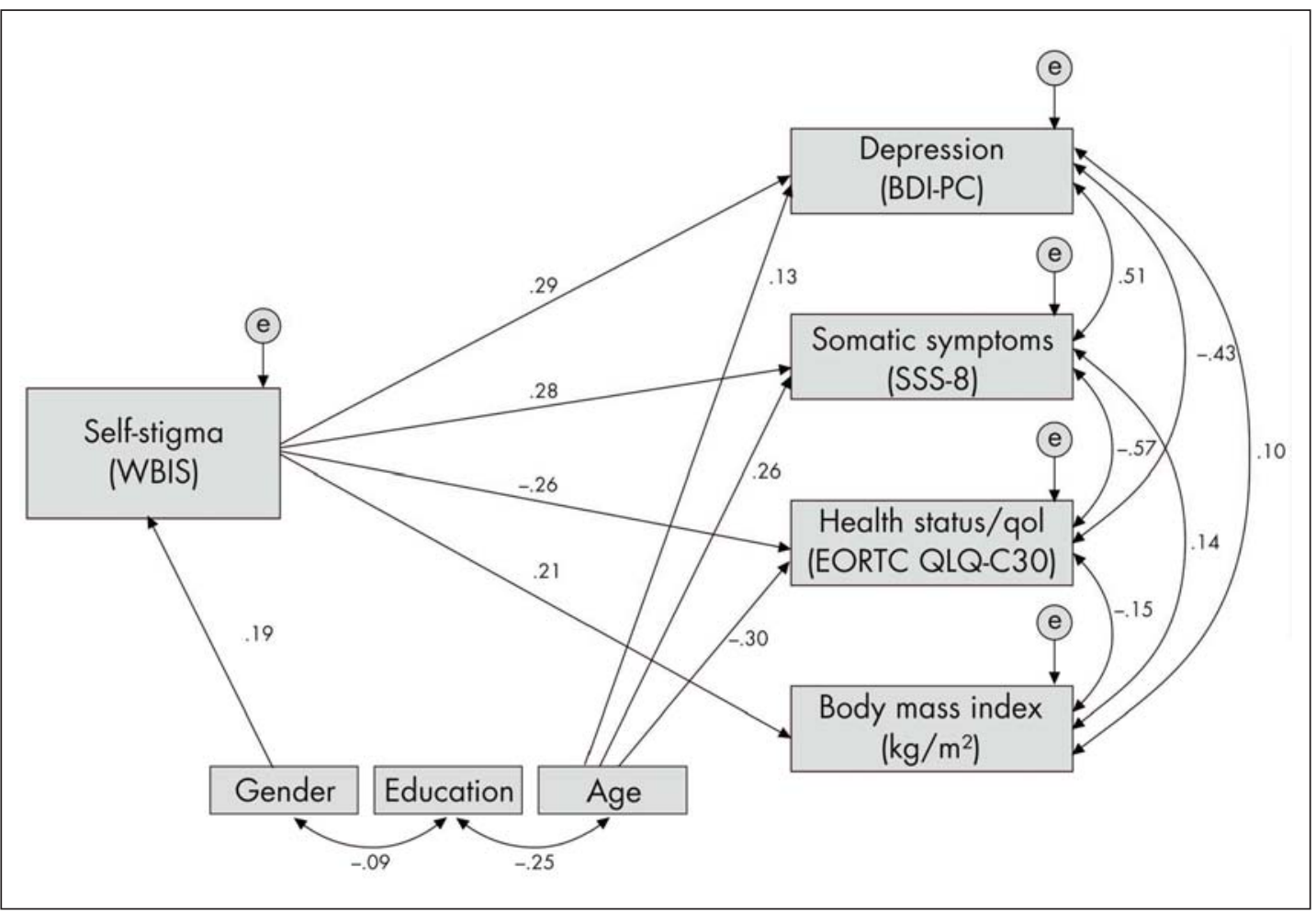

Fig. 1. Predictive effects of self-stigma on mental and physical health outcomes. Notes: Standardized regression coefficients are depicted. All coefficients are significant at $\mathrm{p}<0.001$. WBIS $=$ Weight Bias Internalization Scale (1-7*, scores indicating less favorable conditions are asterisked); BDI-PC, Beck Depression Inventory for Primary Care $\left(0-21^{*}\right)$; SCS = Self-Compassion Scale $\left(1^{*}-5\right)$; SSS-8 = Somatic Symptom Score-8 (0-32*); EORTC QLQ-C30 = European Organization for Research and Treatment of Cancer Quality of Life Questionnaire - Core $30\left(0^{*}-100\right)$.

The results of structural equation modeling are presented in table 2; both models showed good model fit. In Model 1, self-stigma significantly predicted greater depression, somatic symptoms, BMI, and lower health status / quality of life (fig. 1; small to medium effects). All outcome variables were significantly associated with each other, with the highest correlations observed among depression, somatic symptoms, and health status / quality of life (large effects). Regarding sociodemographic variables, significant associations were found between selfstigma and female gender (small effect), and between higher age and greater somatic symptoms and depression, as well as lower health status / quality of life (small to medium effects).

With the inclusion of self-compassion in the model (Model 2), associations between selfstigma and outcome variables changed substantially (fig. 2). Self-compassion partially mediated the relationship between self-stigma (medium effect) and the outcome variables of depression, somatic symptoms, and health status / quality of life (small to medium effects), lowering the association between self-stigma and these outcome variables by approximately one-third. The relationship between self-stigma and BMI, however, was not mediated by selfcompassion.

Post-hoc analyses for significant associations revealed differential mediation effects of SCS subscales. SCS self-judgment, isolation, and over-identification, similar to the SCS total score, partially mediated the effect of self-stigma on health outcomes. Specifically, low selfjudgment, isolation, and over-identification (i.e. high scores on these subscales) were asso- 
Hilbert et al.: Self-Compassion as a Resource in the Self-Stigma Process of Overweight and Obese Individuals

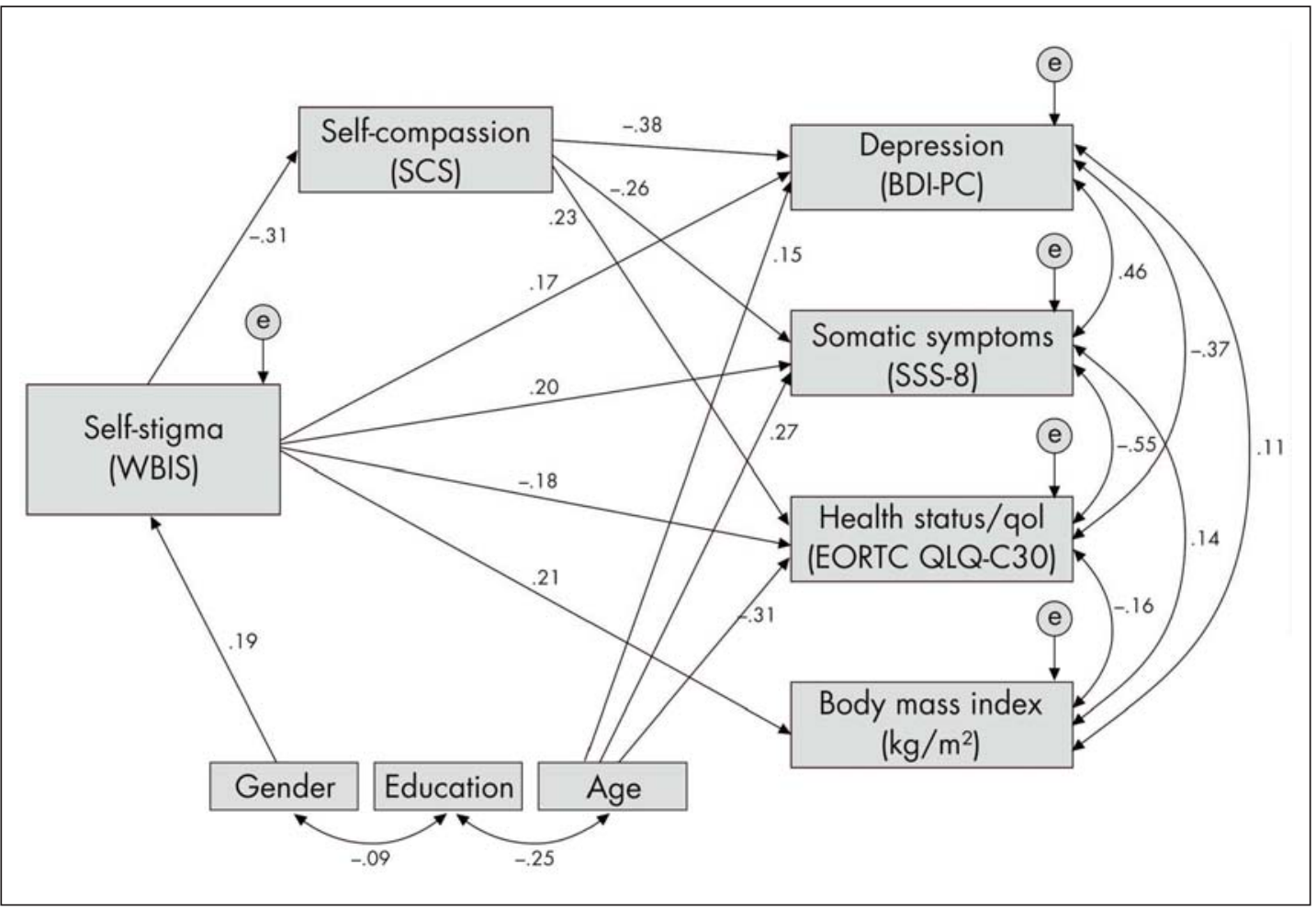

Fig. 2. Self-compassion as a mediator of the association between self-stigma and mental and physical health outcomes. Notes: Standardized regression coefficients are depicted. All coefficients are significant at $\mathrm{p}<$ 0.001. WBIS = Weight Bias Internalization Scale (1-7*, scores indicating less favorable conditions are asterisked); BDI-PC = Beck Depression Inventory for Primary Care (0-21*); SCS = Self-Compassion Scale (1*-5); SSS-8 = Somatic Symptom Score-8 (0-32*); EORTC QLQ-C30 = European Organization for Research and Treatment of Cancer Quality of Life Questionnaire - Core $30\left(0^{*}-100\right)$.

ciated with lower levels of self-stigma (path coefficients -0.33 to $-0.25, \mathrm{p}<0.001$ ), depression and somatic symptoms $(-0.38$ to $-0.27, \mathrm{p}<0.001)$, and higher levels of health status / quality of life $(0.20$ to $0.31, p<0.001)$, lowering the association between self-stigma and health outcomes from small- to medium-size to small-size prediction effects. Among these SCS subscales, low isolation was found to have greatest mediation effects, exceeding those of the SCS total score. Regarding positive self-compassionate aspects, only SCS self-kindness was significantly associated with both self-stigma $(-0.08, p=0.009)$ and all health outcomes $(-0.12$ to $0.06, p<0.05)$, but did not serve as a mediator on the effect of self-stigma on health outcomes. SCS mindfulness was significantly negatively associated with self-stigma $(-0.09$, $\mathrm{p}=0.004)$ and depression only $(-0.09, \mathrm{p}=0.002)$, but did not show any mediation effects on the association between self-stigma and depression. SCS common humanity was not significantly associated with either self-stigma or health outcomes (all $p>0.05$ ).

\section{Discussion}

This demonstration of self-compassion, based on the SCS total score, as a partial mediator of the association between self-stigma and mental and global health outcomes suggests that self-compassion represents a psychological resource, lowering the effect of self-stigma on 
Hilbert et al.: Self-Compassion as a Resource in the Self-Stigma Process of Overweight and Obese Individuals

depression, somatic symptoms, and health status / quality of life by approximately one-third. Thus, self-compassion has the potential to act as a buffer against the mental and global health detriments of self-stigma in overweight and obesity. The mediator effect was independent from sociodemographic factors, although it may be more relevant for women, who reported a higher level of self-stigma than men. In contrast to the mediating effect on mental and global health outcomes, self-compassion was unrelated to BMI and did not mediate the association between self-stigma and BMI. Other related resources that are more directly linked to weight management, for example, mindful eating behavior [27], may be more likely to account for lowering the association between self-stigma and BMI than general self-compassion.

Specifically, the protective effect of self-compassion was based on low levels of selfisolating and self-judgmental attitudes as well as on low over-identification with negative thoughts and feelings. These results suggested that less engagement in dysfunctional cognitive processes may buffer against adverse effects of self-stigma in overweight and obese individuals. In contrast, the mere presence of positive self-compassion that represents general attitudes to be self-kind, self-attentive, and to take personal experiences into greater perspective did not act as a resource in the psychopathology-prone self-stigma process. Among the positive self-compassionate aspects, self-kindness and mindfulness revealed small and mostly negative associations with self-stigma and health outcomes. For clinical implications, these results suggest that compassion-focused therapies, originally designed for people high in shame and self-criticism to cultivate an attitude of inner kindness toward personal failure and distress [39], could be promising for fostering functional self-stigma processing in overweight and obese individuals [40,41]. In contrast, while mindfulnessbased interventions are effective for the treatment of eating behaviors related to weight gain and obesity (e.g., external and emotional eating) [38], their protective effects regarding psychopathological outcomes of self-stigma may be less pronounced.

Interpretation of the results is limited by the self-report assessment of height and weight, used to determine BMI, overweight, and obesity. Self-report commonly leads to an underestimation of height and weight [42]. Strengths of this study include the representative sample, a response rate comparable to that of other surveys, and the use of validated self-report instruments.

Overall, this study provides an initial indication that self-compassion can be a psychological resource in the self-stigma process of overweight and obese individuals as it negatively relates to adverse mental and global health consequences. Thus, self-compassion could represent a target for interventions to reduce self-stigma and to prevent negative, obesityassociated mental and global health outcomes [43]. As self-compassion was found to be associated with lower levels of stress-induced inflammation [44], the effects of self-compassion on the physical sequelae of obesity warrant further investigation.

\section{Acknowledgements}

This research was supported by internal funds by the Department of Medical Psychology and Medical Sociology at the University of Leipzig and by grant 01E01001 from the German Federal Ministry of Education and Research. Funding mechanisms had no role in study design, data collection, analysis and interpretation of data, decision to publish, or writing of the manuscript. We are grateful to Jamie L. Manwaring, Ph.D., Carolyn Edwards, B.Sc., and Barbara Brendel for their editing of the current paper.

\section{Disclosure Statement}

The authors declare that they have no competing interests. 
Hilbert et al.: Self-Compassion as a Resource in the Self-Stigma Process of Overweight and Obese Individuals

\section{References}

1 Lewis M: Shame and stigma; in Gilbert P, Andrews B (eds): Shame. Interpersonal Behavior, Psychopathology, and Culture. New York, Oxford University Press, 1998, pp 126-140.

2 Goffman E: Stigma: Notes on the Management of Spoiled Identity. New York, Simon and Schuster, 1986.

-3 Sikorski C, Luppa M, Braehler E, Koenig H-H, Riedel-Heller SG: Obese children, adults and senior citizens in the eyes of the general public: results of a representative study on stigma and causation of obesity. PloS ONE 2012; 7:e46924.

4 Puhl RM, King KM: Weight discrimination and bullying. Best Pract Res Clin Endocrinol Metab 2013;27:117127.

5 Corrigan PW, Watson AC, Barr L: The self-stigma of mental illness: implications for self-esteem and selfefficacy. J Soc Clin Psychol 2006;25:875-884.

6 Corrigan PW, Watson AC: The paradox of self-stigma and mental illness. Clin Psychol Sci Prac 2002;9:35-53.

7 Puhl RM, Moss-Racusin CA, Schwartz MB: Internalization of weight bias: implications for binge eating and emotional well-being. Obesity 2007;15:19-23.

8 Schwartz MB, Vartanian LR, Nosek BA, Brownell KD: The influence of one's own body weight on implicit and explicit anti-fat bias. Obesity 2006;14:440-447.

-9 Goss K, Allan S: Shame, pride and eating disorders. Clin Psychol Psychother 2009;16:303-316.

10 Masheb RM, Grilo CM, Brondolo E: Shame and its psychopathologic correlates in two women's health problems: binge eating disorder and vulvodynia. Eat Weight Disord Stud 1999;4:187-193.

11 Hilbert A, Braehler E, Haeuser W, Zenger M: Weight bias internalization, core self-evaluation, and health in overweight and obese persons. Obesity 2014;22:79-85.

12 Carels RA, Wott CB, Young KM, Gumble A, Koball A, Oehlhof MW: Implicit, explicit, and internalized weight bias and psychosocial maladjustment among treatment-seeking adults. Eat Behav 2010;11:180-185.

13 Durso LE, Latner JD: Understanding self-directed stigma: development of the weight bias internalization scale. Obesity 2008;16(suppl 2):S80-S86.

14 Durso LE, Latner JD, White MA, Masheb RM, Blomquist KK, Morgan PT, Grilo CM: Internalized weight bias in obese patients with binge eating disorder: associations with eating disturbances and psychological functioning. Int J Eat Disord 2012;45:423-427.

15 Hilbert A, Baldofski S, Zenger M, Kersting A, Braehler E: Weight Bias Internalization Scale: psychometric properties and population norms. PLoS ONE 2014;29:e86303.

16 Lillis J, Luoma JB, Levin ME, Hayes SC: Measuring weight self-stigma: the weight self-stigma questionnaire. Obesity 2010;18:971-976.

17 Crocker J, Major B: Social stigma and self-esteem: the self-protective properties of stigma. Psychol Rev 1989; 96:608.

18 Camp DL, Finlay WML, Lyons E: Is low self-esteem an inevitable consequence of stigma? An example from women with chronic mental health problems. Soc Sci Med 2002;55:823-834.

19 Neff KD: The development and validation of a scale to measure self-compassion. Self Identity 2003;2:223-250.

20 Kelly AC, Vimalakanthan K, Miller KE: Self-compassion moderates the relationship between body mass index and both eating disorder pathology and body image flexibility. Body Image 2014;11:446-453.

-21 MacBeth A, Gumley A: Exploring compassion: a meta-analysis of the association between self-compassion and psychopathology. Clin Psychol Rev 2012;32:545-552.

22 Wren AA, Somers TJ, Wright MA, Goetz MC, Leary MR, Fras AM, Huh BK, Rogers LL, Keefe FJ: Self-compassion in patients with persistent musculoskeletal pain: relationship of self-compassion to adjustment to persistent pain. J Pain Symptom Manage 2012;43:759-770.

-23 Ferreira C, Pinto-Gouveia J, Duarte C: Self-compassion in the face of shame and body image dissatisfaction: implications for eating disorders. Eat Behav 2013;14:207-210.

24 Webb JB, Forman MJ: Evaluating the indirect effect of self-compassion on binge eating severity through cognitive-affective self-regulatory pathways. Eat Behav 2013;14:224-228.

-25 Schoenefeld SJ, Webb JB: Self-compassion and intuitive eating in college women: Examining the contributions of distress tolerance and body image acceptance and action. Eat Behav 2013;14:493-496.

26 Wasylkiw L, MacKinnon AL, MacLellan AM: Exploring the link between self-compassion and body image in university women. Body Image 2012;9:236-245.

27 Mantzios M, Wilson JC: Making concrete construals mindful: a novel approach for developing mindfulness and self-compassion to assist weight loss. Psychol Health 2014;29:422-441.

28 Godsey J: The role of mindfulness based interventions in the treatment of obesity and eating disorders: an integrative review. Complement Ther Med 2013;21:430-439.

29 Marchiori D, Papies EK: A brief mindfulness intervention reduces unhealthy eating when hungry, but not the portion size effect. Appetite 2014;75:40-45.

-30 Hupfeld J, Ruffieux N: Validation of a German version of the Self-Compassion Scale (SCS-D). Z Klin Psychol Psychother 2011;40:115-123.

-31 Gierk B, Kohlmann S, Kroenke K, Spangenberg L, Zenger M, Brahler E, Löwe B: The Somatic Symptom Scale-8 (SSS-8): a brief measure of somatic symptom burden. JAMA Intern Med 2014;174:399-407.

-32 Beck AT, Guth D, Steer RA, Ball R: Screening for major depression disorders in medical inpatients with the Beck Depression Inventory for Primary Care. Behav Res Ther 1997;35:785-791. 
Hilbert et al.: Self-Compassion as a Resource in the Self-Stigma Process of Overweight and Obese Individuals

-33 Kliem S, Mößle T, Zenger M, Braehler E: Reliability and validity of the Beck Depression Inventory-Fast Screen for medical patients in the general German population. J Aff Disord 2014;156:236-239.

-34 Aaronson NK, Ahmedzai S, Bergman B, Bullinger M, Cull A, Duez NJ, Filiberti A, Flechtner H, Fleishman SB, de Haes JC, Kaasa S, Klee M, Osoba D, Rofe PB, Schraub S, Sneeuw K, Sullivan M, Takeda F: The European Organization for Research and Treatment of Cancer QLQ-C30: a quality-of-life instrument for use in international clinical trials in oncology. J Natl Cancer Inst 1993;85:365-376.

-35 Schwarz R, Hinz A: Reference data for the quality of life questionnaire EORTC QLQ-C30 in the general German population. Eur J Cancer 2001;37:1345-1351.

36 Schermelleh-Engel K, Moosbrugger H, Mueller H: Evaluating the fit of structural equation models: tests of significance and descriptive goodness-of-fit measures. Meth Psychol Res 2003;8:23-74.

37 Cohen J: Statistical Power Analysis for the Behavioral Sciences, 2nd ed. Hillsdale, Erlbaum, 1988.

38 O’Reilly GA, Cook L, Spruijt-Metz D, Black DS: Mindfulness-based interventions for obesity-related eating behaviours: a literature review. Obes Rev 2014;15:453-461.

-39 Gilbert P, Procter S: Compassionate mind training for people with high shame and self-criticism: overview and pilot study of a group therapy approach. Clin Psychol Psychother 2006;13:353-379.

40 Gale C, Gilbert P, Read N, Goss K: An evaluation of the impact of introducing compassion focused therapy to a standard treatment programme for people with eating disorders. Clin Pychol Psychother 2014;21:1-12.

41 Kelly AC, Carter JC, Borairi S: Are improvements in shame and self-compassion early in eating disorders treatment associated with better patient outcomes? Int J Eat Disord 2014;47:54-64.

-42 Visscher TL, Viet AL, Kroesbergen IH, Seidell JC: Underreporting of BMI in adults and its effect on obesity prevalence estimations in the period 1998 to 2001. Obesity 2006;14:2054-2063.

43 Danielsdottir S, O’Brien KS, Ciao A: Anti-fat prejudice reduction: a review of published studies. Obes Facts 2010;3:47-58.

44 Breines JG, Thoma MV, Gianferante D, Hanlin L, Chen X, Rohleder N: Self-compassion as a predictor of interleukin-6 response to acute psychosocial stress. Brain Behav Immun 2014;37:109-114. 